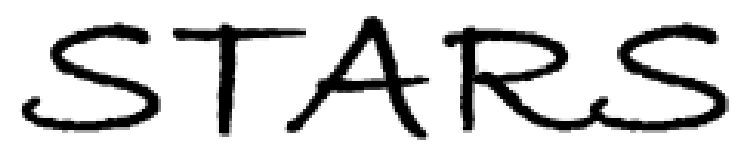

University of Central Florida

STARS

$1-1-2004$

\title{
Normally black reflective twisted-nematic cell for microdisplay application
}

Xinyu Zhu

University of Central Florida

Shin-Tson Wu

University of Central Florida

Find similar works at: https://stars.library.ucf.edu/facultybib2000 University of Central Florida Libraries http://library.ucf.edu

This Article is brought to you for free and open access by the Faculty Bibliography at STARS. It has been accepted for inclusion in Faculty Bibliography 2000s by an authorized administrator of STARS. For more information, please contactSTARS@ucf.edu.

\section{Recommended Citation}

Zhu, Xinyu and Wu, Shin-Tson, "Normally black reflective twisted-nematic cell for microdisplay application" (2004). Faculty Bibliography 2000s. 4925.

https://stars.library.ucf.edu/facultybib2000/4925

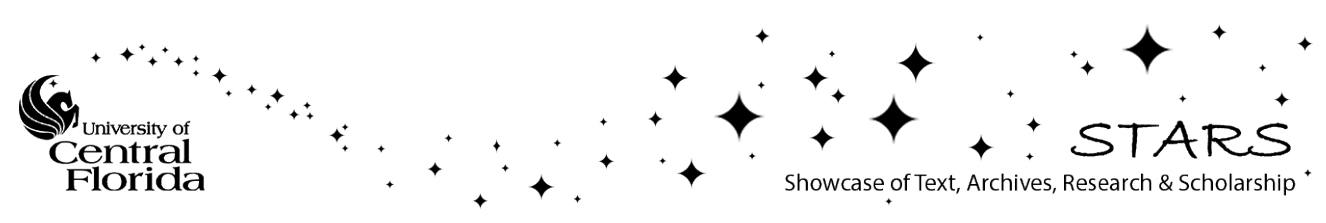




\section{Normally black reflective twisted-nematic cell for microdisplay application}

Cite as: Journal of Applied Physics 95, 7660 (2004); https://doi.org/10.1063/1.1751234

Submitted: 20 February 2004 . Accepted: 25 March 2004 . Published Online: 04 June 2004

Xinyu Zhu, and Shin-Tson Wu

ARTICLES YOU MAY BE INTERESTED IN

Mixed-mode twisted nematic liquid crystal cells for reflective displays

Applied Physics Letters 68, 1455 (1996); https://doi.org/10.1063/1.116252

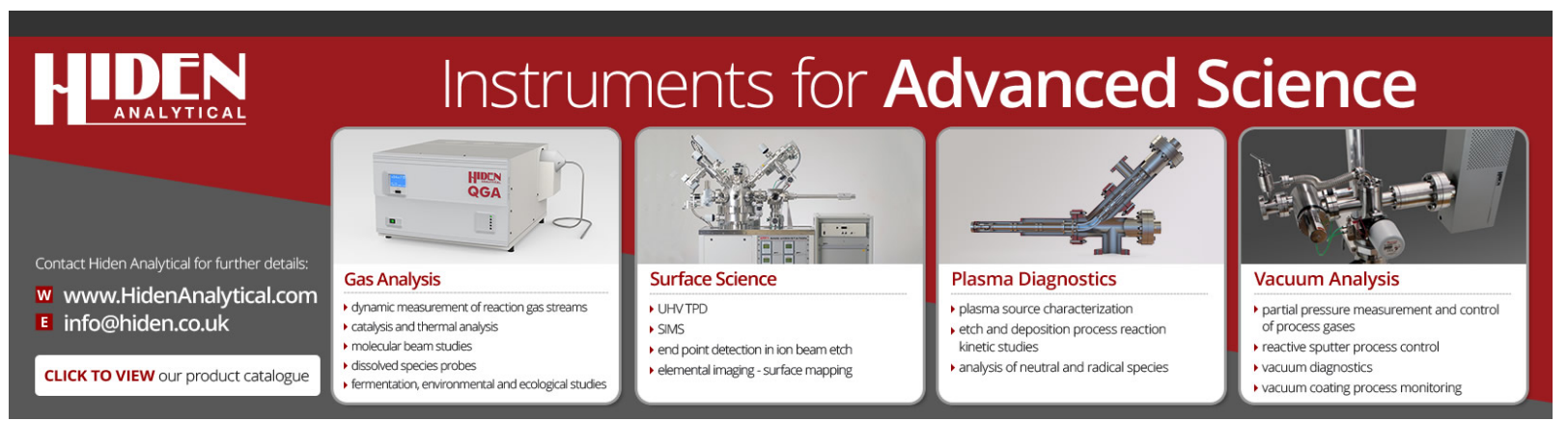




\title{
Normally black reflective twisted-nematic cell for microdisplay application
}

\author{
Xinyu Zhu and Shin-Tson $\mathrm{Wu}^{\mathrm{a})}$ \\ School of Optics, University of Central Florida, Orlando, Florida 32816
}

(Received 20 February 2004; accepted 25 March 2004)

\begin{abstract}
A normally black reflective twisted-nematic mode is proposed for microdisplay application. This mode is based on the polarization rotation effect of a twisted-nematic liquid crystal. In the dark state, the cell exhibits a large gap tolerance and weak color dispersion. For twist angle ranging from $45^{\circ}$ to $65^{\circ}$, the maximum normalized reflectance can achieve $100 \%$ through $d / p$ (cell gap over pitch) ratio optimization. Particularly, when the twist angle equals to $52^{\circ}$, the normalized reflectance can reach $100 \%$ without any chiral dopant. The twist angle and polarizer angle also make important contributions to the bright state optical efficiency and dark state spectral bandwidth. (C) 2004 American Institute of Physics. [DOI: 10.1063/1.1751234]
\end{abstract}

\section{INTRODUCTION}

Liquid crystal on silicon (LCoS) $)^{1,2}$ is an emerging rearprojection microdisplay developed for high definition televisions (HDTVs). Due to the high electron mobility, the pixel size of the LCoS can be made as small as $5-10 \mu \mathrm{m}$. Therefore, for a HDTV resolution $(1920 \times 1080$ pixels $)$, the panel diagonal size is less than $2.2 \mathrm{~cm}$. For such a microdisplay, liquid crystal functions as a light valve. LCoS has been used extensively in front projection, rear projection, and near-theeye virtual projection displays.

Several LC modes have been proposed for LCoS microdisplay applications, such as vertical alignment (VA) mode, ${ }^{3}$ mixed-mode twisted nematic (MTN), ${ }^{4}$ self-compensated twisted nematic mode, ${ }^{5}$ and $45^{\circ}$ reflective twisted-nematic (RTN) mode. ${ }^{6}$ The VA mode exhibits an unprecedented contrast ratio, but it suffers from a serious fringing field effect from adjacent pixels. ${ }^{7-9}$ As a result, each bright pixel is split into two unequal parts separated by a dark line. This split pixel decreases the optical efficiency to $\sim 70 \%$. The $90^{\circ}$ MTN cell is a normally white display using both polarization rotation and birefringence effects, therefore exhibiting a high contrast ratio and weak color dispersion. Moreover, its fringing field effect is the weakest among all of the available LC modes developed for microdisplay applications. ${ }^{9}$ A drawback is that its maximum normalized reflectance is limited to $\sim 88 \%$. On the other hand, the $45^{\circ} \mathrm{RTN}$ cell is a normally black display. ${ }^{6}$ Its cell gap is in the $4-5 \mu \mathrm{m}$ range, depending on the LC birefringence employed. Large cell gap helps to enhance manufacturing yield. Moreover, the on-state voltage for the $45^{\circ}$ RTN cell is relatively low. Without chiral additive, its normalized reflectance is limited to $\sim 95 \%$. The major shortcoming of the $45^{\circ}$ cell is that its fringing field effect is quite severe which degrades the device contrast ratio and optical efficiency. A simple method to suppress the fringing field effect is to use a thinner cell gap. If the cell gap is below $3 \mu \mathrm{m}$, the fringing field effect is reduced substantially.

\footnotetext{
a) Author to whom correspondence should be addressed; electronic mail: swu@mail.ucf.edu
}

In this article, we present a normally black $52^{\circ} \mathrm{RTN}$ mode for microdisplay applications. This mode provides a better overall performance over the $45^{\circ} \mathrm{RTN}$ mode. Detailed simulation results are presented in Sec. II.

\section{LC MODE}

In a $\mathrm{TN}$ reflective display, the cell gap and birefringence product $(d \Delta n)$ is large so that the polarization rotation effect is the major light modulation mechanism. ${ }^{10}$ In the voltage-off state, the LC directors are uniformly twisted from the bottom to the top substrate. The incident linearly polarized light basically follows the rotation of the TN LC, provided that $d \Delta n \gg \lambda$ is satisfied. If such a TN LC is used in reflective display, the reflected beam preserves the same polarization as the incident linearly polarized light due to the pure polarization rotation effect. Therefore, in a microdisplay system incorporating a polarizing beam splitter (PBS), the reflected light from the LCoS panel is blocked by the PBS resulting in a dark state. Such a RTN-based microdisplay is usually operated in the normally black mode.

Figure 1 shows the structure of a RTN based microdisplay. It consists of a reflective TN cell and a PBS. If the incident polarization axis of the PBS makes an angle $\beta$ with respect to the top substrate $\mathrm{LC}$ alignment direction, then the normalized reflectance $\left(R_{\perp}\right)$ can be expressed as ${ }^{11}$

$$
R_{\perp}=\left(\Gamma \frac{\sin X}{X}\right)^{2}\left(\sin 2 \beta \cos X-\frac{\phi}{X} \cos 2 \beta \sin X\right)^{2},
$$

where $\Gamma=2 \pi d \Delta n / \lambda$ and $X=\sqrt{\phi^{2}+(\Gamma / 2)^{2}}$. Here $d$ is the cell gap, $\Delta n$ is the LC birefringence, $\lambda$ is the incident wavelength, and $\phi$ is the twist angle of the TN LC. We define the left-handed twist angle as positive while the right-handed twist as negative.

From Eq. (1), if $\sin X=0$, then $R_{\perp}=0$; independent of $\beta$ angle. Apparently, this will lead to the Gooch-Tarry minimum conditions ${ }^{12}$

$$
\sqrt{\phi^{2}+(\Gamma / 2)^{2}}=m \pi \quad(m=1,2,3 \ldots) .
$$

Figure 2 plots the $d \Delta n / \lambda$ dependent $R_{\perp}$ at different twist angles with $\beta=0^{\circ}$. As expected, there exists a series of 


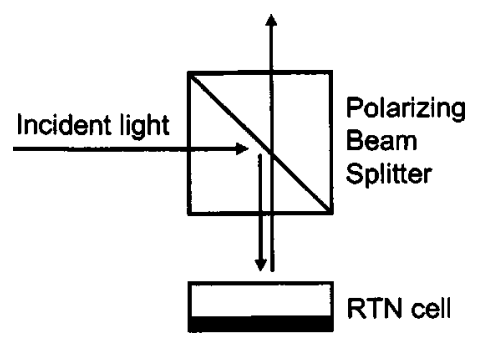

FIG. 1. Schematic view of a RTN mode microdisplay.

Gooch-Tarry minima. From the response time viewpoint, a small cell gap is preferred. Thus, the first minimum condition $(m=1)$ is commonly chosen for RTN mode. From Fig. 2, the $90^{\circ}$ RTN does not lead to a high reflectance bright state when the effective $d \Delta n / \lambda$ is reduced (i.e., voltage is increased). The normalized reflectance $R_{\perp}$ reaches $100 \%$ when the twist angle is around $60^{\circ}$. However, this is under the assumption that LC directors are uniformly twisted throughout the cell. When the applied voltage exceeds a threshold, the LC directors are tilted up so that the assumption of uniform twist does not hold anymore. To determine the optimal twist angle, the voltage-on state needs to be calculated.

\section{A. LC parameters}

In order to calculate the electro-optic properties of a RTN mode with applied voltage, we first use the continuum elastic theory ${ }^{13}$ to obtain the LC director distributions and then use the extended Jones matrix method ${ }^{14}$ to calculate the optical reflectance. In this article, we use Merck MLC-6694100 LC mixture for the numerical simulations. The relevant LC parameters are listed as follows: $K_{11}=13.9 \mathrm{pN}, K_{22}$ $=9.0 \mathrm{pN}, K_{33}=21.5 \mathrm{pN}, \epsilon_{\|}=10.9, \epsilon_{\perp}=3.5, n_{e}=1.5925$, and $n_{o}=1.4865$ at $\lambda=589 \mathrm{~nm}$ and $T=20{ }^{\circ} \mathrm{C}$. During simulations, the LC birefringence dispersion ${ }^{15}$ for the red-greenblue (RGB) colors has also been taken into consideration.

\section{B. Effect of $d / p$ ratio}

For a TN cell, a small amount of chiral dopant is added in order to control the twist sense. By changing the concentration of chiral dopant the pitch length $(p)$ can be adjusted. To study the LC pitch length effect on the bright state reflec-

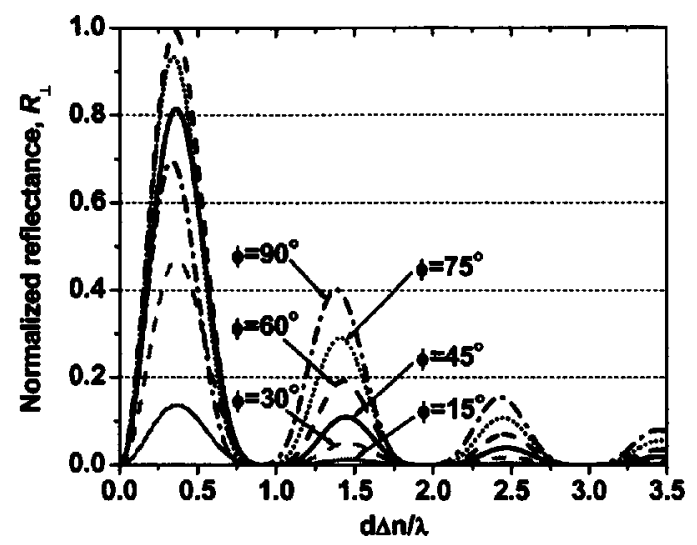

FIG. 2. Dependence of $R_{\perp}$ on $d \Delta n / \lambda$ at different twist angles for $\beta=0^{\circ}$.

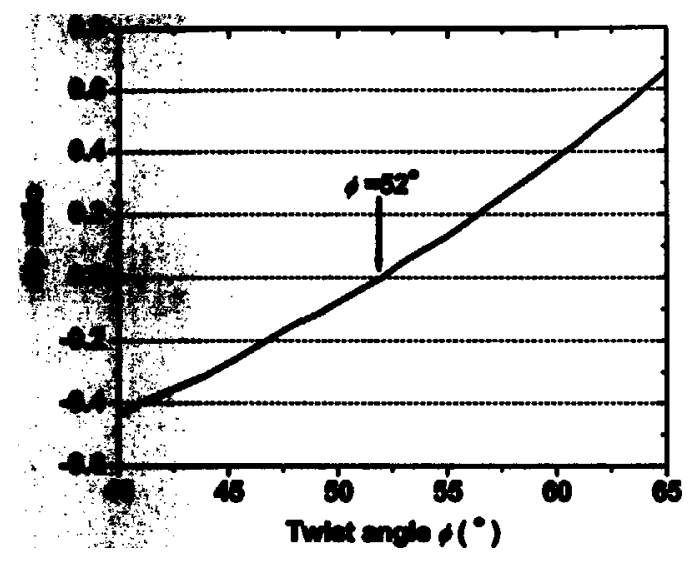

FIG. 3. The $d / p$ ratio required to achieving $R_{\perp}=1$ at different twist angles for $\beta=0^{\circ}$ and $\lambda=550 \mathrm{~nm}$.

tance, we scan the $d / p$ ratio for a given twist angle. The cell gap is predetermined by Eq. (2) for the first minimum condition $(m=1)$. For every $d / p$ ratio, the voltage dependent normalized reflectance is calculated and the maximum value selected. We then choose the $d / p$ value which corresponds to $R_{\perp}=1$ for each twist angle.

Figure 3 depicts the $d / p$ ratio required for achieving $R_{\perp}=1$ for the RTN cells at $\beta=0^{\circ}$. As the twist angle increases from $\phi=40^{\circ}$ to $65^{\circ}$, the required $d / p$ ratio also increases gradually from negative to positive. A negative $d / p$ ratio means the sense of chiral dopant is opposite to the twist sense of the RTN cell. When $\phi=52^{\circ}, d / p=0$, which means no chiral dopant is necessary to obtain $R_{\perp}=1$. This result is consistent with that reported by $\mathrm{Yu}^{16}$ and Beynon, ${ }^{17}$ except that we used crossed polarizers (PBS) instead of parallel polarizers used therein. For $\phi>52^{\circ}$, a left-handed chiral dopant is necessary to boost $R_{\perp}$ to $100 \%$, while a right-handedness chiral dopant is needed for $\phi<52^{\circ}$. In our simulations, we assume the LC layer has a left-handed twist angle.

Figure 4 shows the effect of different $d / p$ ratios on the maximum $R_{\perp}$. Apparently, when $d / p=0$ or $d / p=\phi / 2 \pi$ there exists only one twist angle whose maximum $R_{\perp}$ is $100 \%$. Nevertheless, if the $d / p$ ratio is optimized as shown in Fig. $3, R_{\perp}=1$ can always be achieved for any twist angle between $40^{\circ}$ and $65^{\circ}$. Of course, an overdosed chiral dopant is unfavorable because it will increase the LC response time.

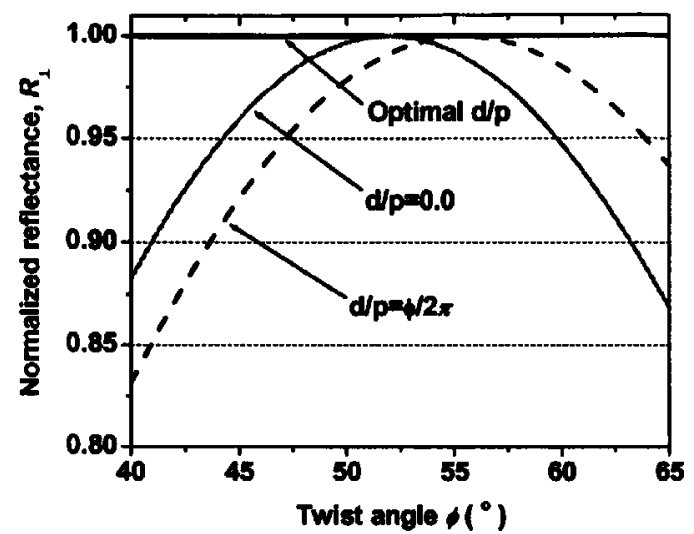

FIG. 4. Effect of $d / p$ ratio on the maximum $R_{\perp}$ for $\beta=0^{\circ}$ and $\lambda=550 \mathrm{~nm}$. 


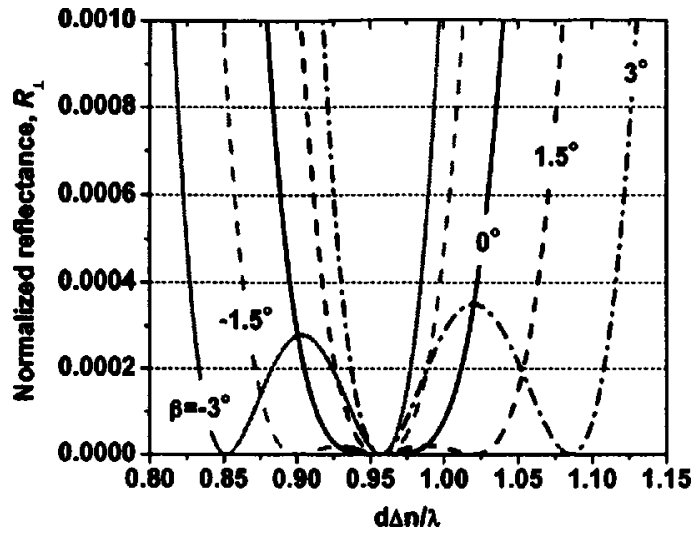

FIG. 5. Dependence of $R_{\perp}$ on $d \Delta n / \lambda$ for different $\beta$ at $\phi=52^{\circ}$.

On the other hand, a negative chiral dopant (i.e., opposite to the LC twist sense) will cause instability to the LC twist structure.

\section{C. $\beta$ angle effect}

To achieve high contrast ratio, a good dark state is critically important. Since the RTN mode is normally black, its dark state is affected by the twist angle $(\phi)$, the $d \Delta n$ value of the LC layer, the $\beta$ angle, and the spectral bandwidth. Figure 5 depicts the $\beta$ angle effect on the dark state reflectance of the $\phi=52^{\circ}$ RTN cell. As long as $\beta \neq 0^{\circ}$, the curves exhibit a "W" shape, which contributes to widen the $d \Delta n / \lambda$ tolerance. On the other hand, the twist angle $\phi$ also affects the dark state $d \Delta n / \lambda$ tolerance when $\beta \neq 0^{\circ}$, as shown in Figs. 6(a) and 6(b). A larger twist angle has a lower light leakage on the central peak of the $\mathrm{W}$ curves, which means a larger twist angle has a larger $\beta$ angle tolerance.

\section{Spectral sensitivity}

For projection display, each primary spectral band has about 50-60 nm bandwidth. To evaluate the spectral sensitivity of the $52^{\circ}$ RTN cell, we plot the voltage-dependent reflectance at red $(620-680 \mathrm{~nm})$, green $(530-570 \mathrm{~nm})$, and blue $(420-480 \mathrm{~nm})$ bands. Results are shown in Fig. 7. In each band, we calculated the reflectance in every $10 \mathrm{~nm}$ step and then took the spectrum average. For these calculations, $\beta$ angle is set at $0^{\circ}$, cell gap $d=4.9 \mu \mathrm{m}$, and $d / p=0$. The dark states of these three colors do not overlap due to the different $d \Delta n / \lambda$ requirement. For instance, a red band needs a larger $d \Delta n$ value in order to obtain a good dark state. Therefore, the three-panel configuration projection system needs to be used in order to achieve high contrast ratio. To obtain a different $d \Delta n$ value for compensating the RGB wavelength difference, Merck has developed the so-called two-bottle LC mixtures. These two LC bottles have nearly the same physical properties (e.g., phase transition temperatures, dielectric constants, and elastic constants) except for different $\Delta n$. By properly mixing the two bottles, three mixtures with different $\Delta n$ values for the RGB channels can be obtained fairly easily.

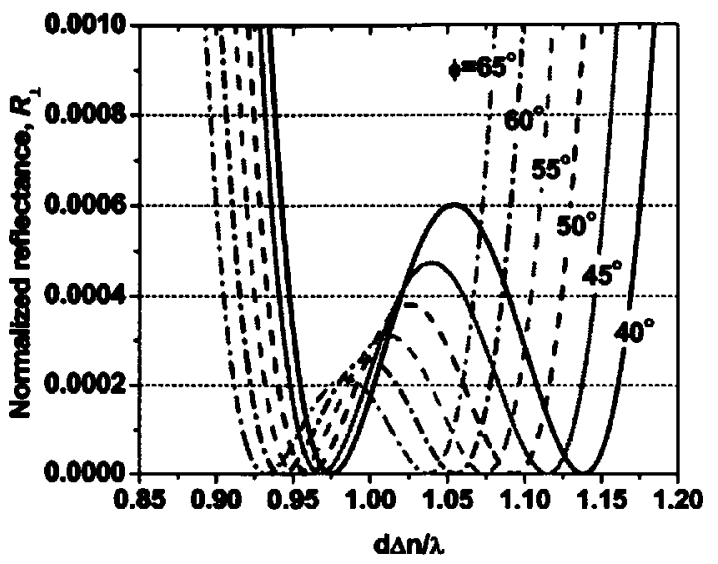

(a)

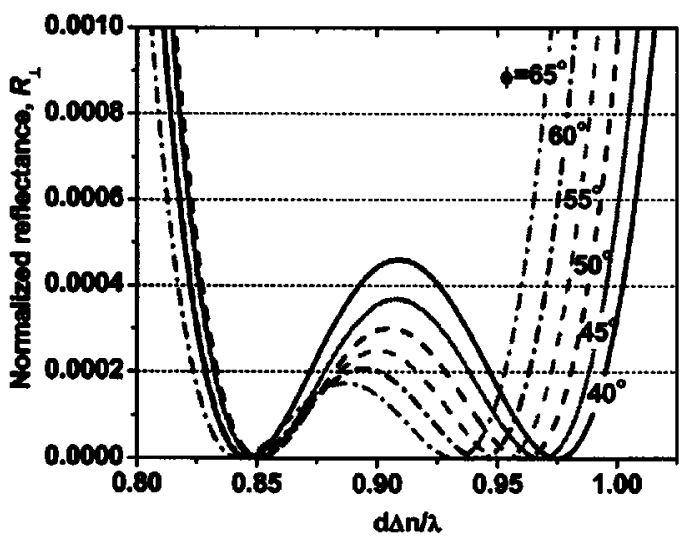

(b)

FIG. 6. Dependence of $R_{\perp}$ on $d \Delta n / \lambda$ for different $\phi$ at: (a) $\beta=3^{\circ}$ and (b) $\beta=-3^{\circ}$.

\section{E. Cell gap tolerance}

Cell gap tolerance is particularly important for the normally black display. Figure 8 shows the cell gap tolerance of the $52^{\circ}$ RTN mode for the green band. Within $\pm 5 \%$ cell gap error, a good dark state is preserved and the contrast ratio maintains larger than 1400:1 $\left(V_{\text {on }}=2.7 \mathrm{~V}_{\text {rms }}\right.$ and $V_{\text {off }}$ $\left.=1.2 \mathrm{~V}_{\mathrm{rms}}\right)$. Large cell gap tolerance also implies to small

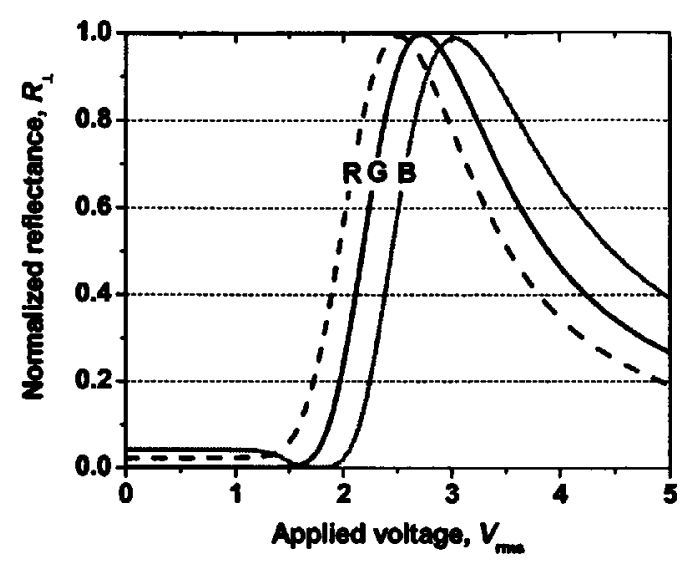

FIG. 7. Voltage dependent reflectance of the $52^{\circ}$ RTN cell at $\beta=0^{\circ}: R$ $=620-680 \mathrm{~nm}, G=530-570 \mathrm{~nm}$, and $B=420-480 \mathrm{~nm}$. 


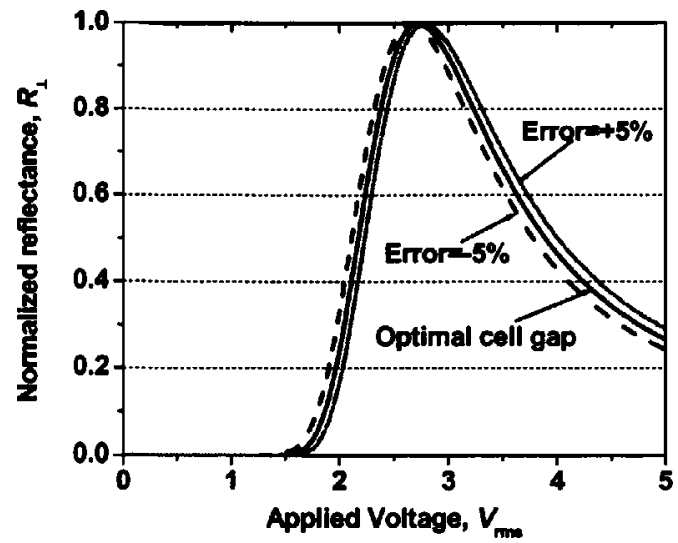

FIG. 8. Cell gap tolerance on the voltage-dependent reflectance of the $52^{\circ}$ RTN cell at $\beta=0^{\circ}$. The simulated contrast ratio for $G=530-570 \mathrm{~nm}$ is 28 000:1 for the optimized cell gap, drops to $5300: 1$ for $+5 \%$ cell gap error, and 1400:1 for $-5 \%$ cell gap error, respectively. Here, $V_{\text {on }}=2.7 \mathrm{~V}_{\text {rms }}$ and $V_{\text {off }}=1.2 \mathrm{~V}_{\mathrm{rms}}$ are assumed.

color dispersion. From Figs. 7 and 8, the on-state voltage is less than $3 \mathrm{~V}_{\mathrm{rms}}$, which is lower than that of VA and MTN modes. For the purpose of comparison, Fig. 9 plots the voltage-dependent reflectance curves of the $52^{\circ} \mathrm{RTN}$ cell and the widely used $45^{\circ}$ RTN cell with and without chiral dopant. Obviously, the on-state reflectance of the $45^{\circ}$ RTN without chiral dopant does not reach $100 \%$. With a suitable dopant $(d / p=-0.27)$, the reflectance can be boosted to $100 \%$. In the mean time, the on-state voltage is slightly decreased due to the pitch length effect. Although the doped chiral agent improves the optical efficiency of the $45^{\circ}$ RTN cell from $\sim 95 \%$ to $100 \%$, it narrows the device viewing cone slightly. Viewing angle of the LC panel is an important issue for LCoS based microdisplay. If the LC panel has a wider viewing cone, then a smaller $F$-number projection lens can be used. As a result, the display brightness is greatly improved. ${ }^{18}$ Thus, the tradeoff for improving $5 \%$ optical efficiency by adding chiral dopant which, in turn, leads to a narrower viewing cone is not worth taking.

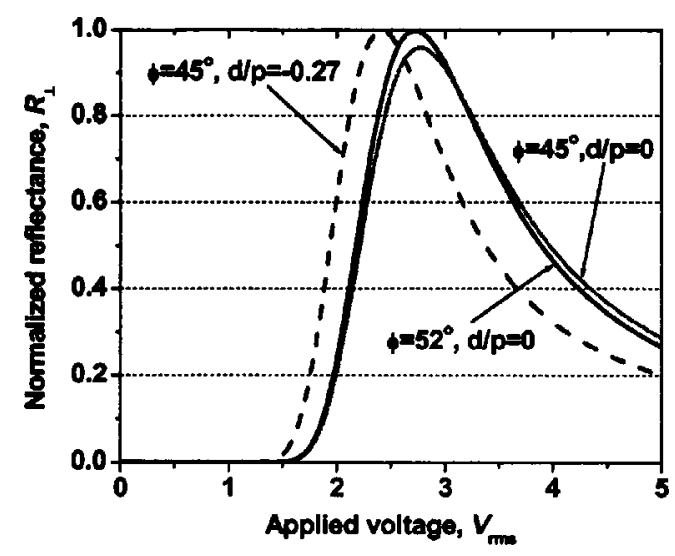

FIG. 9. Voltage dependent reflectance of the $45^{\circ}$ and $52^{\circ}$ RTN cells at $\beta=0^{\circ}$. Incident light is green band $\lambda=530-570 \mathrm{~nm}$.

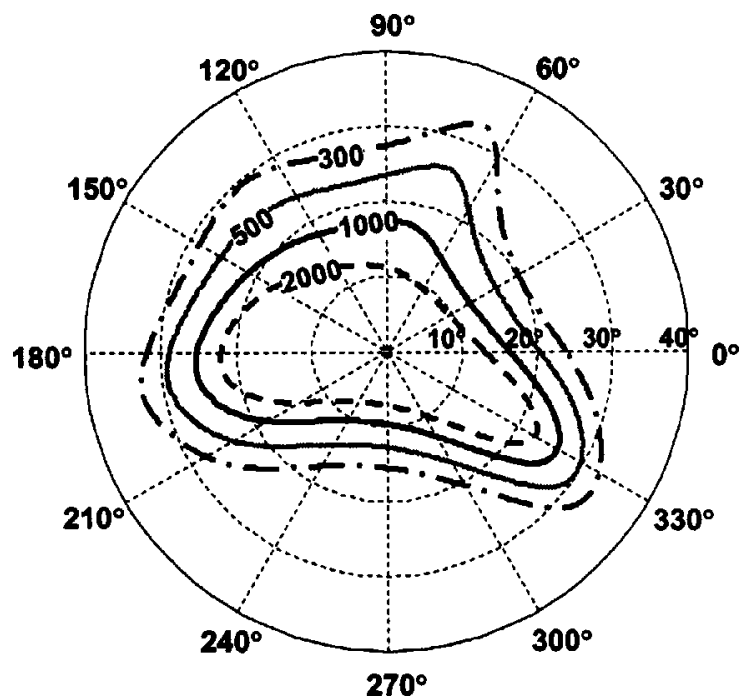

FIG. 10. Iso-contrast contour of the $52^{\circ}$ RTN cell with $V_{\text {on }}=2.7 \mathrm{~V}_{\text {rms }}, V_{\text {off }}$ $=1.2 \mathrm{~V}_{\mathrm{rms}}$, and $\lambda=530-570 \mathrm{~nm}$.

\section{F. View angle}

A wide viewing cone allows a smaller $F$-number projection lens to be used for enhancing display brightness. Figure 10 shows the simulated iso-contrast contours of the $52^{\circ}$ RTN cell. During simulations, the on-state voltage is assumed at $2.7 \mathrm{~V}_{\text {rms }}$, dark state voltage at $1.2 \mathrm{~V}_{\text {rms }}$, and the incident green light has a bandwidth covering $\lambda=530-570 \mathrm{~nm}$. From Fig. 10, the 1000:1 contrast ratio is achieved within the central $10^{\circ}$ viewing cone. A typical $F$-number for the projection lens is about 2.5-3.0.

\section{CONCLUSION}

A normally black $52^{\circ} \mathrm{RTN}$ mode exhibits a high contrast ratio, high light efficiency, low driving voltage, and large cell gap tolerance. By properly optimizing the $d / p$ ratio, the normalized reflectance can reach $100 \%$ for any twist angle from $40^{\circ}$ to $65^{\circ}$. A higher twist angle has better polarizer angle tolerance and a lower twist angle has lower color dispersion at the dark state. The $52^{\circ}$ RTN mode will find potential application in LCoS based microdisplay.

\section{ACKNOWLEDGMENTS}

This work is partially supported by AFOSR under Contract No. F49620-01-1-0377 and Toppoly Optoelectronics Corporation.

${ }^{1}$ P. M. Alt, Conference Record of the Int'l Display Research Conference, 1997, M19-28.

${ }^{2}$ H. Kurogane, K. Doi, T. Nishihata, A. Honma, M. Furuya, S. Nakagaki, and I. Takanashi, Soc. Inf. Display Tech. Digest 29, 33 (1998).

${ }^{3}$ M. F. Schiekel and K. Fahrenschon, Appl. Phys. Lett. 19, 391 (1971).

${ }^{4}$ S. T. Wu and C. S. Wu, Appl. Phys. Lett. 68, 1455 (1996).

${ }^{5}$ K. H. Yang, Proceedings Europe Display '96, 1996, p. 449.

${ }^{6}$ J. Grinberg, A. Jacobson, W. Bleha, L. Miller, I. Fraas, D. Bosewell, and G. Myer, Opt. Eng. (Bellingham) 14, 217 (1975).

${ }^{7}$ Y. Ji, J. Gandhi, and M. Stefanov, Soc. Inf. Display Tech. Digest 30, 750 (1999).

${ }^{8}$ H. De Smet, J. Van den Steen, and A. Van Calster, Soc. Inf. Display Tech. Digest 32, 968 (2001). 
${ }^{9}$ K. H. Fan Chiang, S. T. Wu, and S. H. Chen, Jpn. J. Appl. Phys., Part 141 , 4577 (2002).

${ }^{10}$ M. Schadt and W. Helfrich, Appl. Phys. Lett. 18, 127 (1971).

${ }^{11}$ S. T. Wu and D. K. Yang, Reflective Liquid Crystal Displays (Wiley, New York, 2001).

${ }^{12}$ C. H. Gooch and H. A. Tarry, J. Phys. D 8, 1575 (1975).

${ }^{13}$ P. G. de Gennes and J. Prost, The Physics of Liquid Crystals, 2nd ed. (Clarendon, Oxford, 1993).
${ }^{14}$ A. Lien, Appl. Phys. Lett. 57, 2767 (1990).

${ }^{15}$ S. T. Wu, Phys. Rev. A 33, 1270 (1986).

${ }^{16}$ F. H. Yu, J. Chen, S. T. Tang, and H. S. Kwok, J. Appl. Phys. 82, 5287 (1997).

${ }^{17}$ E. Beynon, K. Saynor, M. Tillin, and M. Towler, J. Soc. Inf. Disp. 7, 71 (1999).

${ }^{18}$ M. L. Jepsen, M. J. Ammer, M. Bolotski, J. J. Drolet, A. Gupta, Y. Lai, D. Huffman, H. Shi, and C. Vieri, Displays 23, 109 (2002). 